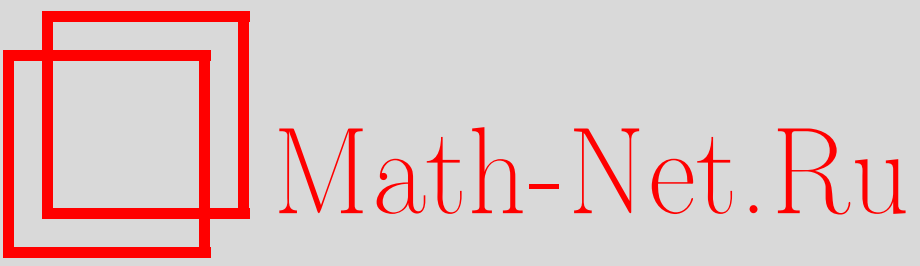

Д. И. Борисов, Р. Р. Гадыльшин, О спектре оператора Шредингера с быстроосциллирующим финитным потенциалом, ТМФ, 2006, том 147, номер 1, 58-63

DOI: https://doi.org/10.4213/tmf2022

Использование Общероссийского математического портала Math-Net.Ru подразумевает, что вы прочитали и согласны с пользовательским соглашением

http://www.mathnet.ru/rus/agreement

Параметры загрузки:

IP : 3.82 .47 .9

26 апреля 2023 г., 07:21:04 
ТЕОРЕТИЧЕСКАЯ

И МАТЕМАТИЧЕСКАЯ

ФИЗИКА

Том 147, № 1

апрель, 2006

(C) 2006 г.

\author{
Д. И. Борисов* , Р. Р. Гадыльшин*
}

\title{
О СПЕКТРЕ ОПЕРАТОРА ШРЕДИНГЕРА С БЫСТРООСЦИЛЛИРУЮЩИМ ФИНИТНЫМ ПОТЕНЦИАЛОМ
}

\begin{abstract}
Изучается эффект возникновения собственного значения из границы существенного спектра оператора Шредингера, возмущенного финитным быстроосциллирующим потенциалом. Доказаны достаточные условия существования и отсутствия такого собственного значения. В случае существования собственного значения получены первые члены его асимптотического разложения.
\end{abstract}

Ключевые слова: оператор Шредингера, спектр, возмущение, асимптотика.

Настоящая работа посвящена изучению спектра оператора

$$
H_{\varepsilon}:=-\frac{d^{2}}{d x^{2}}+V\left(x, \frac{x}{\varepsilon}\right)
$$

в $L_{2}(\mathbb{R})$ с областью определения $W_{2}^{2}(\mathbb{R})$. Здесь $\varepsilon$ - малый положительный параметр, $V(x, \xi)$ - комплекснозначная 1-периодическая по $\xi$ функция из $C^{\infty}\left(\mathbb{R}^{2}\right)$ такая, что для всех $\xi \in \mathbb{R}$ носители $\operatorname{supp} V(\cdot, \xi)$ ограничены равномерно по $\xi$.

Оператор $H_{0}:=-d^{2} / d x^{2}$ в $L_{2}(\mathbb{R})$ с областью определения $W_{2}^{2}(\mathbb{R})$ самосопряжен, его дискретный спектр пуст, существенный спектр совпадает с полуосью $[0,+\infty)$, а оператор умножения на функцию $V(x, x / \varepsilon)$ является $H_{0}$-компактным. Поэтому в силу теорем 1.1, 5.35 главы IV монографии [1] оператор $H_{\varepsilon}$ замкнут для всех $\varepsilon$, и существенные спектры операторов $H_{\varepsilon}$ и $H_{0}$ совпадают.

Целью работы является изучение вопроса о существовании и асимптотическом поведении собственных значений оператора $H_{\varepsilon}$, стремящихся к нулю при $\varepsilon \rightarrow 0$, в случае, когда для всех $x \in \mathbb{R}$ среднее значение функции $V(x, \cdot)$ по периоду равно нулю. Такие собственные значения можно также рассматривать как возникающие из границы существенного спектра при возмущении оператора $H_{0}$ потенциалом $V(x, x / \varepsilon)$. Отметим, что эффект возникновения собственных значений из границы существенного спектра при возмущении потенциалом исследовался в работах [2] для

*Башкирский государственный педагогический университет, Уфа, Россия. E-mail: BorisovDI@ic.bashedu.ru, BorisovDI@yandex.ru, gadylshin@bspu.ru, gadylshin@yandex.ru 
потенциалов вида $\varepsilon U(x)$, где $U(x)$ - достаточно быстро убывающий вещественный потенциал. Подобный эффект исследовался и в [3] для возмущения вида $\varepsilon L_{\varepsilon}$, где $L_{\varepsilon}: W_{2, \text { lос }}^{2}(\mathbb{R}) \rightarrow L_{2}(\mathbb{R} ; Q)$ - произвольный линейный оператор, удовлетворяющий равномерному по $\varepsilon$ неравенству $\left\|L_{\varepsilon} u\right\|_{L_{2}(\mathbb{R})} \leqslant C\|u\|_{W_{2}^{2}(Q)}$ для некоторого конечного интервала $Q$ на оси, а $L_{2}(\mathbb{R} ; Q)$ - подмножество функций из $L_{2}(\mathbb{R})$ с носителями из $\bar{Q}$. Для указанных возмущений в [2], [3] был изучен вопрос о существовании собственных значений, возникающих из границы непрерывного спектра, а в случае существования были построены первые члены асимптотического разложения данного собственного значения. Отметим также, что эффект возникновения собственных значений из существенного спектра в случае малых возмущений исследовался и в работах [4]. Возмущения, рассмотренные в цитированных работах, не включают в себя потенциал $V(x, x / \varepsilon)$. Более того, возмущение, описываемое таким потенциалом, не является регулярным в том смысле, что оператор умножения на этот потенциал при $\varepsilon \rightarrow 0$ не стремится равномерно к нулю.

Пусть отрезок $M=\left[x_{0}, x_{1}\right]$ таков, что для всех $\xi \in \mathbb{R}$ выполнено условие $\operatorname{supp} V(\cdot, \xi) \subseteq M$. Через $W$ обозначим множество 1-периодических по $\xi$ функций $u(x, \xi)$ из $C^{\infty}\left(\mathbb{R}^{2}\right)$ таких, что $\operatorname{supp} u(\cdot, \xi) \subseteq M$ для всех $\xi \in \mathbb{R}$. Для функций из $W$ среднее по периоду обозначим как

$$
\langle u(x, \cdot)\rangle:=\int_{0}^{1} u(x, \xi) d \xi .
$$

Лемма. Для любой функции $u \in W$ и любого числа $n \geqslant 1$ верно равенство

$$
\int_{\mathbb{R}} u\left(x, \frac{x}{\varepsilon}\right) d x=\int_{\mathbb{R}}\langle u(x, \cdot)\rangle d x+O\left(\varepsilon^{n}\right) .
$$

ДоКАЗАТЕЛЬСтво. Утверждение леммы достаточно доказать для случая $\langle u(x, \cdot)\rangle \equiv 0$, так как общий случай сводится к данному заменой $\tilde{u}(x, \xi)=u(x, \xi)-$ $\langle u(x, \cdot)\rangle$. Пусть $\langle u(x, \cdot)\rangle \equiv 0$. Введем обозначение

$$
P[u](x, \xi):=\int_{0}^{\xi} u(x, \tau) d \tau+\int_{0}^{1} \tau u(x, \tau) d \tau .
$$

Тогда $P[u] \in W$ и $\langle P[u](x, \cdot)\rangle=0$. Учитывая очевидное равенство

$$
u\left(x, \frac{x}{\varepsilon}\right)=\varepsilon \frac{d}{d x} P[u]\left(x, \frac{x}{\varepsilon}\right)-\varepsilon \frac{\partial P[u]}{\partial x}\left(x, \frac{x}{\varepsilon}\right),
$$

получаем

$$
\int_{\mathbb{R}} u\left(x, \frac{x}{\varepsilon}\right) d x=-\varepsilon \int_{\mathbb{R}} \frac{\partial P[u]}{\partial x}\left(x, \frac{x}{\varepsilon}\right) d x .
$$

Так как $\frac{\partial P[u]}{\partial x} \in W$ и $\left\langle\frac{\partial P[u]}{\partial x}(x, \cdot)\right\rangle \equiv 0$, то равенство (1) применимо и к функции $\frac{\partial P[u]}{\partial x}$. Применяя это равенство достаточное число раз, приходим к утверждению леммы. 
Обозначим

$$
k_{2}:=\frac{1}{2} \int_{\mathbb{R}}\left\langle(P[V](x, \cdot))^{2}\right\rangle d x .
$$

Основным результатом работы является следующая

TEOPEMA. Пyсms

$$
\langle V(x, \cdot)\rangle \equiv 0
$$

Тогда имеют место следующие утверждения.

1. Если $\operatorname{Re} k_{2}>0$, то существует единственное собственное значение $\lambda_{\varepsilon}$ оператора $H_{\varepsilon}$, стремящееся $\kappa$ нулю при $\varepsilon \rightarrow 0$. Данное собственное значение простое, $u$ его асимптотика имеет вид

$$
\lambda_{\varepsilon}=-\varepsilon^{4} k_{2}^{2}+O\left(\varepsilon^{5}\right) .
$$

2. Если $\operatorname{Re} k_{2}<0$, то оператор $H_{\varepsilon}$ не имеет собственных значений, стремящихся $\kappa$ нулю при $\varepsilon \rightarrow 0$.

ДокАзАтЕЛьСтво. Легко проверить, что функция

$$
v(x, \xi):=\int_{0}^{\xi}(\xi-\tau) V(x, \tau) d \tau+\xi \int_{0}^{1} \tau V(x, \tau) d \tau+v_{1}(x)
$$

является решением уравнения

$$
\frac{\partial^{2} v}{\partial \xi^{2}}=V
$$

причем

$$
\frac{\partial v}{\partial \xi}=P[V]
$$

В силу условия (3) и принадлежности $V \in W$ функция $v$ является 1-периодической по $\xi$, и, следовательно, ограниченной равномерно по $(x, \xi) \in \mathbb{R}^{2}$. Будем считать, что $v_{1} \in C^{\infty}(\mathbb{R})$ и $\operatorname{supp} v_{1} \subseteq M$, тогда $v \in W$.

Положим $q_{\varepsilon}(x, \xi):=1+\varepsilon^{2} v(x, \xi)$. Оператор умножения на функцию $\tilde{q}_{\varepsilon}(x):=$ $q_{\varepsilon}(x, x / \varepsilon)$ (обозначим его через $Q_{\varepsilon}$ ) взаимно однозначно отображает $L_{2}(\mathbb{R})$ на себя. Поэтому собственные значения оператора $H_{\varepsilon}$ совпадают с собственными значениями оператора $Q_{\varepsilon}^{-1} H_{\varepsilon} Q_{\varepsilon}$. В силу (5) и определения $\tilde{q}_{\varepsilon}(x)$ справедливо представление $Q_{\varepsilon}^{-1} H_{\varepsilon} Q_{\varepsilon}=H_{0}-\varepsilon L_{\varepsilon}$, где

$$
L_{\varepsilon}=\varepsilon \frac{2}{\tilde{q}_{\varepsilon}(x)} \frac{d}{d x} v\left(x, \frac{x}{\varepsilon}\right) \frac{d}{d x}-\frac{f_{\varepsilon}\left(x, \frac{x}{\varepsilon}\right)}{\tilde{q}_{\varepsilon}(x)}, \quad f_{\varepsilon}=\varepsilon V v-\varepsilon \frac{\partial^{2} v}{\partial x^{2}}-2 \frac{\partial^{2} v}{\partial x \partial \xi} .
$$

Так как $V, v \in W$, то носители коэффициентов оператора $L_{\varepsilon}$ лежат в $M$ для всех значений $\varepsilon$, а оператор $L_{\varepsilon}: W_{2, \text { loc }}^{2}(\mathbb{R}) \rightarrow L_{2}(\mathbb{R} ; M)$ удовлетворяет равномерному по $\varepsilon$ неравенству

$$
\left\|L_{\varepsilon} u\right\|_{L_{2}(\mathbb{R})} \leqslant C\|u\|_{W_{2}^{2}(M)} .
$$


Введем обозначения

$$
\begin{gathered}
m_{\varepsilon}^{(1)}=\int_{\mathbb{R}} L_{\varepsilon}[1] d x, \quad m_{\varepsilon}^{(2)}=\int_{\mathbb{R}} L_{\varepsilon}\left[\int_{\mathbb{R}}|x-t| L_{\varepsilon}[1] d t\right] d x, \\
k_{\varepsilon}=\frac{\varepsilon}{2} m_{\varepsilon}^{(1)}+\frac{\varepsilon^{2}}{2} m_{\varepsilon}^{(2)} .
\end{gathered}
$$

Так как для оператора $L_{\varepsilon}$ справедлива оценка (8), то из теоремы 1 работы [3] следует, что если

$$
k_{\varepsilon}=\varepsilon c_{1}+\varepsilon^{2} c_{2}+O\left(\varepsilon^{3}\right), \quad c_{1}, c_{2}=\mathrm{const}
$$

то достаточным условием наличия у оператора $\left(H_{0}-\varepsilon L_{\varepsilon}\right)$ собственного значения, стремящегося к нулю при $\varepsilon \rightarrow 0$, является неравенство $\operatorname{Re}\left(c_{1}+\varepsilon c_{2}\right)>0$, а достаточным условием отсутствия такого собственного значения - неравенство $\operatorname{Re}\left(c_{1}+\right.$ $\left.\varepsilon c_{2}\right)<0$. Если $\operatorname{Re}\left(c_{1}+\varepsilon c_{2}\right)>0$, то оператор $\left(H_{0}-\varepsilon L_{\varepsilon}\right)$ имеет единственное собственное значение, стремящееся к нулю, данное собственное значения является простым, и имеет место равенство $\lambda_{\varepsilon}=-\left(\varepsilon c_{1}+\varepsilon^{2} c_{2}\right)^{2}+O\left(c_{1} \varepsilon^{4}+\varepsilon^{5}\right)$.

Таким образом, для доказательства теоремы достаточно показать справедливость равенства (10) при $c_{1}=0, c_{2}=k_{2}$. Докажем это. Для упрощения вычислений положим

$$
v_{1}(x)=\frac{1}{2} \int_{0}^{1}\left(\tau-\tau^{2}\right) V(x, \tau) d \tau .
$$

Легко видеть, что тогда

$$
\langle v(x, \cdot)\rangle=0
$$

следовательно,

$$
\left\langle\frac{\partial^{2} v}{\partial x^{2}}(x, \cdot)\right\rangle=\left\langle\frac{\partial^{2} v}{\partial x \partial \xi}(x, \cdot)\right\rangle=0 .
$$

Обозначим $\tilde{f}_{\varepsilon}(x):=f_{\varepsilon}(x, x / \varepsilon)$. Из $(9),(7)$ и определения $\tilde{q}_{\varepsilon}$ в силу леммы и равенств $(12),(5),(6)$ получаем, что

$$
\begin{aligned}
\frac{\varepsilon m_{\varepsilon}^{(1)}}{2} & =-\frac{\varepsilon}{2} \int_{\mathbb{R}} \frac{\tilde{f}_{\varepsilon}(x)}{\tilde{q}_{\varepsilon}(x)} d x=-\frac{\varepsilon^{2}}{2} \int_{\mathbb{R}} V\left(x, \frac{x}{\varepsilon}\right) v\left(x, \frac{x}{\varepsilon}\right) d x+O\left(\varepsilon^{3}\right)= \\
& =-\frac{\varepsilon^{2}}{2} \int_{\mathbb{R}}\langle V(x, \cdot) v(x, \cdot)\rangle d x+O\left(\varepsilon^{3}\right)=-\frac{\varepsilon^{2}}{2} \int_{\mathbb{R}}\left\langle v(x, \cdot) \frac{\partial^{2} v}{\partial \xi^{2}}(x, \cdot)\right\rangle d x+O\left(\varepsilon^{3}\right)= \\
& =\frac{\varepsilon^{2}}{2} \int_{\mathbb{R}}\left\langle\left(\frac{\partial v}{\partial \xi}(x, \cdot)\right)^{2}\right\rangle d x+O\left(\varepsilon^{3}\right)=\varepsilon^{2} k_{2}+O\left(\varepsilon^{3}\right),
\end{aligned}
$$

где $k_{2}$ определено в формуле (2).

Так как в силу определения $\tilde{q}_{\varepsilon}$

$$
\frac{d}{d x} \ln \tilde{q}_{\varepsilon}(x)=\frac{\varepsilon^{2}}{\tilde{q}_{\varepsilon}(x)} \frac{d}{d x} v\left(x, \frac{x}{\varepsilon}\right),
$$


то, интегрируя по частям и учитывая, что для любой функции $g \in C_{0}(\mathbb{R})$ справедливо равенство

$$
\frac{d^{2}}{d x^{2}} \int_{\mathbb{R}}|x-t| g(t) d t=2 g(x)
$$

из $(9),(7)$ получаем

$$
\begin{aligned}
\frac{\varepsilon^{2} m_{\varepsilon}^{(2)}}{2} & =-\varepsilon \int_{\mathbb{R}} \ln \tilde{q}_{\varepsilon}(x) \frac{d^{2}}{d x^{2}} \int_{\mathbb{R}}|x-t| \frac{\tilde{f}_{\varepsilon}(t)}{\tilde{q}_{\varepsilon}(t)} d t d x+\frac{\varepsilon^{2}}{2} \int_{M^{2}} \frac{|x-t| \tilde{f}_{\varepsilon}(x) \tilde{f}_{\varepsilon}(t)}{\tilde{q}_{\varepsilon}(x) \tilde{q}_{\varepsilon}(t)} d t d x= \\
& =-2 \varepsilon \int_{\mathbb{R}} \frac{\tilde{f}_{\varepsilon}(x) \ln \tilde{q}_{\varepsilon}(x)}{\tilde{q}_{\varepsilon}(x)} d x+\varepsilon^{2} \int_{x_{0}}^{x_{1}} \frac{\tilde{f}_{\varepsilon}(x)}{\tilde{q}_{\varepsilon}(x)}\left(\int_{x_{0}}^{x} \frac{(x-t) \tilde{f}_{\varepsilon}(t)}{\tilde{q}_{\varepsilon}(t)} d t\right) d x
\end{aligned}
$$

Первое слагаемое в правой части последнего равенства есть величина порядка $O\left(\varepsilon^{3}\right)$. Интегрируя по частям второе слагаемое, запишем цепочку равенств

$$
\begin{aligned}
\int_{x_{0}}^{x_{1}} \frac{\tilde{f}_{\varepsilon}(x)}{\tilde{q}_{\varepsilon}(x)} & \left(\int_{x_{0}}^{x} \frac{(x-t) \tilde{f}_{\varepsilon}(t)}{\tilde{q}_{\varepsilon}(t)} d t\right) d x= \\
= & \int_{x_{0}}^{x_{1}} x \tilde{f}_{\varepsilon}(x)\left(\int_{x_{0}}^{x} \tilde{f}_{\varepsilon}(t) d t\right) d x-\int_{x_{0}}^{x_{1}} \tilde{f}_{\varepsilon}(x)\left(\int_{x_{0}}^{x} t \tilde{f}_{\varepsilon}(t) d t\right) d x+O\left(\varepsilon^{2}\right)= \\
= & 2 \int_{x_{0}}^{x_{1}} x \tilde{f}_{\varepsilon}(x)\left(\int_{x_{0}}^{x} \tilde{f}_{\varepsilon}(t) d t\right) d x-\int_{\mathbb{R}} x \tilde{f}_{\varepsilon}(x) d x \int_{\mathbb{R}} \tilde{f}_{\varepsilon}(x) d x+O\left(\varepsilon^{2}\right)= \\
= & -\int_{x_{0}}^{x_{1}}\left(\int_{x_{0}}^{x} \tilde{f}_{\varepsilon}(t) d t\right)^{2} d x+\int_{\mathbb{R}}\left(x_{1}-x\right) \tilde{f}_{\varepsilon}(x) d x \int_{\mathbb{R}} \tilde{f}_{\varepsilon}(x) d x+O\left(\varepsilon^{2}\right) .
\end{aligned}
$$

Из определения функции $\tilde{f}_{\varepsilon}$, формулы для $f_{\varepsilon}$ из $(7)$, равенств $(12)$ и леммы следует, что второе слагаемое в правой части последнего равенства есть величина порядка $O\left(\varepsilon^{2}\right)$. Вновь учитывая определение функции $\tilde{f}_{\varepsilon}$ и равенство

$$
\frac{\partial^{2} v}{\partial x \partial \xi}\left(x, \frac{x}{\varepsilon}\right)=\varepsilon \frac{d}{d x} \frac{\partial v}{\partial x}\left(x, \frac{x}{\varepsilon}\right)-\varepsilon \frac{\partial^{2} v}{\partial x^{2}}\left(x, \frac{x}{\varepsilon}\right)
$$

получаем

$$
-\int_{x_{0}}^{x_{1}}\left(\int_{x_{0}}^{x} \tilde{f}_{\varepsilon}(t) d t\right)^{2} d x=-4 \int_{M}\left(\int_{x_{0}}^{x} \frac{\partial^{2} v}{\partial x \partial \xi}\left(t, \frac{t}{\varepsilon}\right) d t\right)^{2} d x+O\left(\varepsilon^{2}\right)=O\left(\varepsilon^{2}\right) .
$$

Таким образом,

$$
\int_{x_{0}}^{x_{1}} \frac{\tilde{f}_{\varepsilon}(x)}{\tilde{q}_{\varepsilon}(x)}\left(\int_{x_{0}}^{x} \frac{(x-t) \tilde{f}_{\varepsilon}(t)}{\tilde{q}_{\varepsilon}(t)} d t\right) d x=O\left(\varepsilon^{2}\right),
$$

а потому $\varepsilon^{2} m_{\varepsilon}^{(2)}=O\left(\varepsilon^{4}\right)$, и равенство (10) с $c_{1}=0, c_{2}=k_{2}$ теперь следует из (9) и $(13)$.

Из теоремы 1 работы [3] также следует, что, кроме собственного значения, стремящегося к нулю, остальные собственные значения оператора $H_{\varepsilon}$ (если они существуют) должны стремиться к бесконечности при $\varepsilon \rightarrow 0$. Если $V$ - вещественнозначная функция (не равная тождественно нулю), то все собственные значения оператора $H_{\varepsilon}$ 
вещественны и отрицательны. Более того, в этом случае $k_{2}$ вещественно и положительно, а потому оператор $H_{\varepsilon}$ имеет единственное собственное значение, данное собственное значение простое, стремится к нулю и имеет асимптотику (4).

Благодарности. Работа выполнена при частичной финансовой поддержке Программы поддержки ведущих научных школ (грант НШ-1446.2003.1) и программы "Университеты России" (грант УР.04.01.484), а также при финансовой поддержке РФФИ (гранты 05-01-97912-р_агидель (Д. И. Борисов) и 05-01-01008 (Р. Р. Гадыльшин)).

\section{Список литературы}

[1] Т. Като, Теория возмущений линейных операторов, Мир, М., 1972.

[2] B. Simon, Ann. Phys., 97 (1976), 279; M. Klaus, Ann. Phys., 108 (1977), 288;

R. Blankenbecler, M. L. Goldberger, B. Simon, Ann. Phys., 108 (1977), 69; M. Klaus, B. Simon, Ann. Phys., 130 (1980), 251.

[3] Р. Р. Гадыльшин, ТМФ, 132:1 (2002), 97-104.

[4] Д. Е. Пелиновский, К. Салем, ТМФ, 122 (2000), 118-128; P. Zhevandrov, A. Merzon, "Asymptotics of eigenfunctions in shallow potential well and related problems", Asymptotic Methods for Wave and Quantum Problems. AMS Transl. Ser. 2. Adv. Math. Sci, ed. M. V. Karasev, AMS, Providence, RI, 2003, 235-284; F. Bentosela, R. M. Cavalcanti, P. Exner, V. A. Zagrebanov, J. Phys. A, 32 (1999), 3029-3039; T. Weidl, Comm. Part. Diff. Eq., 24:1\&2 (1999), 25-60. 